Journal for ImmunoTherapy of Cancer

\title{
Genomic alterations in biliary tract cancer predict prognosis and immunotherapy outcomes
}

Xiaofeng Chen, ${ }^{1}$ Deqiang Wang, ${ }^{2}$ Jing Liu, ${ }^{3}$ Jingrong Qiu, ${ }^{4}$ Jun Zhou, ${ }^{5}$ Jieer Ying, ${ }^{6}$ Yan Shi, ${ }^{3}$ Zhaoxia Wang, ${ }^{7}$ Haizhou Lou, ${ }^{8}$ Jiuwei Cui, ${ }^{9}$ Jingdong Zhang, ${ }^{10}$ Yunpeng Liu, ${ }^{11,12}$ Fengjiao Zhao, ${ }^{1}$ Lanlan Pan, ${ }^{1}$ Jianyi Zhao (1) , ${ }^{1}$ Dongqin Zhu, ${ }^{13}$ Shiqing Chen, ${ }^{14}$ Xiangcheng Li, ${ }^{15}$ Xue Li, ${ }^{13}$ Liuqing Zhu, ${ }^{13}$ Yang Shao, ${ }^{13,16}$ Yongqian Shu (D) ${ }^{1}$

To cite: Chen X, Wang D, Liu J, et al. Genomic alterations in biliary tract cancer predict prognosis and immunotherapy outcomes. Journal for ImmunoTherapy of Cancer 2021;9:e003214. doi:10.1136/ jitc-2021-003214

- Additional supplemental material is published online only. To view, please visit the journal online (http://dx.doi.org/10. 1136/jitc-2021-003214).

XC, DW, JL and JQ are joint first authors.

Accepted 22 October 2021

Check for updates

(c) Author(s) (or their employer(s)) 2021. Re-use permitted under CC BY-NC. No commercial re-use. See rights and permissions. Published by BMJ.

For numbered affiliations see end of article.

Correspondence to Dr Yongqian Shu;

shuyongqian@csco.org.cn

\section{ABSTRACT}

Background Recently, immunotherapy with immune checkpoint inhibitors (ICls) has shown promising efficacy in biliary tract cancer (BTC), which includes gallbladder cancer (GBC) and cholangiocarcinoma (CHOL). Understanding the association between immunotherapy outcomes and the genomic profile of advanced BTC may further improve the clinical benefits from immunotherapy.

Methods Genomic tumor DNA was isolated from 98 Chinese patients with advanced BTC and used for targeted next-generation sequencing of 416 cancerrelated genes to identify the genomic alterations common to advanced BTC. Thirty-four patients had received ICI camrelizumab plus gemcitabine and oxaliplatin (from the NCT03486678 trial) as a first-line treatment. Tumor-infiltrating immune cells were evaluated using immunofluorescence staining.

Results KRAS and TP53 mutations were much more frequent in the advanced-stage BTC cohort than in other cohorts with mostly early stage disease. Specifically, KRAS-TP53 co-mutations were favored in advanced $\mathrm{CHOL}$, with a favorable response to immunotherapy, while single KRAS mutations predicted poor prognosis and immunotherapy outcomes for CHOL. Compared with $\mathrm{GBC}, \mathrm{CHOL}$ had more mutations in genes involved in KRAS signaling; a high mutation load in these genes correlated with poor immunotherapy outcomes and may subsequently cause inferior immunotherapy outcomes for $\mathrm{CHOL}$ relative to $\mathrm{GBC}$. Furthermore, a genomic signature including 11 genes was developed; their mutated subtype was associated with poor prognosis and immunotherapy outcomes in both $\mathrm{CHOL}$ and GBC. Transcriptome analyses suggested immune dysfunction in the signature mutated subtype, which was validated by tumor microenvironment (TME) evaluation based on detection of immune cell infiltration. Importantly, the signature wild-type subtype with favorable TME may be an advantageous population of immunotherapy.

Conclusions Genomic alterations in advanced BTC were associated with specific prognosis and immunotherapy outcomes. Combining genomic classification with TME evaluation further improved the stratification of immunotherapy outcomes.

\section{BACKGROUND}

Biliary tract cancers (BTCs) include gallbladder cancer (GBC) and cholangiocarcinoma (CHOL). BTG constitutes approximately $3 \%$ of all gastrointestinal malignancies and 150,000 patients were diagnosed with BTC in 2015 worldwide. ${ }^{1}$ Moreover, BTC is an aggressive disease with poor prognosis for which the median overall survival (OS) is less than 1 year and 5-year OS is below $5 \% .^{2}$ Currently, the regimen of gemcitabine plus cisplatin (GemCis) is the standard first-line therapy for advanced BTC. However, BTC is typically resistant to chemotherapy, the objective response rate (ORR) for GemCis is only $26 \%$ and the median OS is just 11.7 months, according to the Advanced Biliary Cancer (ABC)-02 Trial. ${ }^{3}$

The use of immune checkpoint inhibitors (ICIs), including antibodies to programmed cell death-1 (PD-1) and programmed cell death ligand-1 (PD-L1), has produced therapy breakthroughs in various malignancies and has been investigated as a treatment for advanced BTC. Earlier studies have shown that ICIs are less effective in previously treated patients with BTC. ${ }^{4}$ More recently, ICIs in combination with chemotherapy appear to have better efficacy and are well tolerated as a first-line therapy. ${ }^{4}$ Specifically, in a phase II single-arm study (NCT03486678), we evaluated the combination of camrelizumab, an anti-PD-1 antibody, with gemcitabine and oxaliplatin (GEMOX) as first-line treatment in advanced BTC. ${ }^{5}$ In 37 participants, we reported that the ORR and disease control rate were $54 \%$ and $89 \%$, while the median progression-free survival and OS were 6.2 months and 12.1 months, respectively. These findings suggest that ICIs combined with chemotherapy can be an effective alternative 
first-line treatment for advanced BTC, although validation through phase III trials is necessary.

Further improving the efficacy of ICIs for treating cancer is a promising area of immunotherapy research. Identifying biomarkers that predict which patients might most benefit from the use of ICIs requires a comprehensive understanding of the genetic characteristics specific to cancer. Although efforts have been made to reveal the genetic alterations common in BTC and determine their prognostic value using next-generation sequencing (NGS), ${ }^{6}$ most of the patients included in these studies have early stage disease and the genomic landscape in advanced BTC remains to be elucidated. More importantly, the association between genomic characteristics and ICI treatment outcomes in advanced BTC remains unknown. Moreover, recent studies investigating the prognostic role of tumor mutation burden (TMB) in advanced BTC treated with ICIs have led to different conclusions. ${ }^{45}$

In this study, we assessed the genomic profiles of advanced BTC in a Chinese cohort and reported how genomic characteristics may be used to determine disease prognosis and predict ICI treatment outcomes. Moreover, classification based on genomic signature may correlate to differing antitumor immunity and provide a powerful method to stratify clinical outcomes.

\section{METHODS \\ Patients}

We retrospectively screened patients from 10 medical centers led by Jiangsu Province Hospital (JPH) in China who were diagnosed with advanced BTC between March 2015 and August 2019. Patients who received the ICI camrelizumab plus the GEMOX regimen from the NCT03486678 trial conducted by JPH were also screened. The following inclusion criteria were used for all patients: pathological diagnosis of CHOL or GBC; stage IV; and sufficient tumor tissue to allow NGS; treatment-naïve when sampling. This study was performed in accordance with the Declaration of Helsinki and the ethical standards of the institutional research committees. Written informed consent was obtained from all participating patients.

The CHOL cohort from The Cancer Genome Atlas (TCGA) $^{7}$ and a previously reported Shanghai GBC cohort ${ }^{8}$ were used for comparisons, and data were acquired and preprocessed as previously described.$^{9}$

\section{Samples collection and preparation}

Tumor tissue and matched normal specimens were sequenced at a Clinical Laboratory Improvement Amendments-certified genomics laboratory in China. Tissue slides were first analyzed to evaluate tumor content and percentage. Only samples with tumor purity $>20 \%$ on histopathological assessment were eligible for genomic profiling.

\section{Targeted NGS and genetic analysis}

As previously reported, ${ }^{9}$ genomic DNAs were extracted, purified and qualified. DNA libraries were subjected to PCR amplification and purification before targeted enrichment. During library preparation, genomic DNAs from each sample were marked with unique indexes and different libraries were pooled together for enrichment with probes targeting 416 cancer-related genes. Prior to sequencing, the captured libraries were examined for quality and quantity using the KAPA Library Quantification Kit (KAPA Biosystems) by qRT-PCR. The final libraries were then sequenced on Hiseq 4000 platforms (Illumina) to reach a mean coverage depth of $60 \times$ for the normal controls and $150 \times(3000 \times)$ for the tumor tissue (plasma) samples after removal of PCR duplicates.

Original image data were transferred by base calling analysis into raw sequence data. Single nucleotide variants (SNVs) and short insertions/deletions (indels) were identified by VarScan2 with the minimum variant allele frequency threshold set at 0.01 , and a $\mathrm{p}$ value threshold for calling variants set at 0.05 to generate Variant Call Format files. All SNVs/indels were annotated with Annotate Variation (ANNOVAR), and each SNV/indel was manually checked on the Integrative Genomics Viewer. Copy number variation analysis was performed using an inhouse developed pipeline. A fold change threshold of 1.6 and 0.6 in DNA copy number was set as the cut-off for amplification and deletion, respectively.

\section{TMB calculation}

Non-synonymous somatic mutations, including missense, nonsense, splice-site, inframe and frameshift mutations, which may be functional, ${ }^{10}$ were included in our analyses. TMB was calculated as the number of non-synonymous somatic mutations. In addition, some genes encode proteins that are part of the KRAS signaling network in the interaction analysis using the STRING database (https://www.string-db.org/). TMB of these genes was defined as K-TMB.

\section{Immunohistochemistry (IHC)}

PD-L1 expression was determined using a Dako PD-L1 IHC 22C3 pharmDx kit (Agilent Technologies) in combination with the Dako Autostainer Link 48 system (Agilent Technologies). The PD-L1 expression level was evaluated by Tumor Proportion Score (TPS) and TPS $\geq 1$ was identified as positive.

\section{Multiplexed IHC (mIHC) and multispectral imaging}

As described previously, ${ }^{11}$ we used the PANO 7-plex IHC kit (Panovue, Beijing, China) for multiplex immunofluorescence staining, according to the manufacturer's instructions. We adopted anti-HLA-DR (ab92511, Abcam, UK), anti-S100 (ab52642), anti-panCK (CST4545, Cell Signaling Technology, USA), anti-CD56 (CST3576), antiCD8 (CST70306), and anti-CD68 (BX50031, Biolynx, China) for use in mIHC, and distinguished the invasive margin and tumor parenchyma by S100 staining. 
We applied the Mantra System (PerkinElmer, Waltham, Massachusetts, USA) to scan the stained slides and reconstructed section images based on a spectral library for multispectral unmixing. Finally, we quantified immune cells using the inForm image analysis software (PerkinElmer, Waltham, Massachusetts, USA).

\section{Tumor immune dysfunction and exclusion (TIDE) scoring}

We used TIDE, a computational method based on transcriptome, to calculate $\mathrm{T}$ cell dysfunction and exclusion scores, which were further merged as the TIDE score to predict tumor response to ICIs. ${ }^{12}$

\section{Tumor microenvironment (TME) scoring}

As previously described, TME was scored using principal component analysis based on gene expressions associated with different TME phenotypes. ${ }^{11}{ }^{13}$ The $\mathrm{R}$ package of TMEscore is available in GitHub (https://github.com/ DongqiangZeng0808/TMEscore).

\section{Statistical analysis}

The $\chi^{2}$ test, Student's $t$-test, Wilcoxon or Mann-Whitney test was used for intergroup comparison as needed and $\mathrm{p}$ value was calculated using a two-tailed test. Clinical outcomes were previously defined. ${ }^{5}$ Survival curves were plotted using the Kaplan-Meier method and analyzed using the log-rank test. Genes with mutations that were significantly associated with OS were screened using univariate Cox models. Least absolute shrinkage and selection operator (LASSO) was further used to identify mutated genes with the most powerful prognostic role. A significant threshold was set at a value of $p<0.05$. All analyses used SPSS (V.23.0, Chicago, Illinois, USA), R (V.3.6.1) and R Bioconductor packages.

\section{RESULTS}

\section{Patient characteristics}

A total of 98 patients with advanced BTC were included, consisting of 36 patients with GBC and 62 patients with CHOL (table 1). Of the patients with CHOL, 15 had perihilar, 5 had distal, 35 had intrahepatic, and seven had unclear location tumors. The median age was 60.1 years (range, 35-80) and 55.1\% (54/98) were women. At the time of initial diagnosis, $71.4 \%(70 / 98)$ performed at ECOG PS $0-1 \%$ and $41.8 \%(41 / 98)$ had tumors with grade 3 histology. OS data were available for 64 patients, 34 of whom were treated with camrelizumab plus GEMOX (from the NCT03486678 trial) as first-line therapy.

\section{Genomic analyses of BTC}

The analysis process of our study is shown in figure 1 . The mutation profiles were similar for patients treated with camrelizumab and untreated patients (online supplemental figure 1$)$. The most commonly mutated gene in both CHOL (55\%; figure 2A) and GBC (72\%; figure 2B) was TP53. The mutation frequencies of 416 genes in the sequencing panel were compared between the advanced disease cohort, and two cohorts with mostly early stage

\begin{tabular}{|c|c|c|c|}
\hline Characteristics & GBC $(n=36)$ & CHOL (n=62) & All $(n=98)$ \\
\hline \multicolumn{4}{|c|}{ Age at initial diagnosis (years) } \\
\hline Median (range) & $63.3(42-80)$ & $58.3(35-74)$ & $60.1(35-80)$ \\
\hline \multicolumn{4}{|l|}{ Gender } \\
\hline Male & $17(47.2 \%)$ & $27(43.5 \%)$ & $44(44.9 \%)$ \\
\hline Female & $19(52.8 \%)$ & $35(56.5 \%)$ & $54(55.1 \%)$ \\
\hline \multicolumn{4}{|c|}{ ECOG PS at initial diagnosis } \\
\hline $0-1$ & $27(75.0 \%)$ & $43(69.4 \%)$ & $70(71.4 \%)$ \\
\hline $2-3$ & $4(11.1 \%)$ & $12(19.4 \%)$ & $16(16.4 \%)$ \\
\hline Unknown & $5(13.9 \%)$ & $7(11.2 \%)$ & $12(12.2 \%)$ \\
\hline \multicolumn{4}{|l|}{ Histology grade } \\
\hline 2 & $7(19.4 \%)$ & $13(21.0 \%)$ & $20(20.4 \%)$ \\
\hline 3 & $17(47.3 \%)$ & $24(38.7 \%)$ & $41(41.8 \%)$ \\
\hline Unknown & $12(33.3 \%)$ & $25(40.3 \%)$ & $37(37.8 \%)$ \\
\hline \multicolumn{4}{|c|}{ Treatment of first-line immunotherapy* } \\
\hline Yes & $12(33.3 \%)$ & $22(35.5 \%)$ & $34(34.7 \%)$ \\
\hline No & $24(66.7 \%)$ & 40 (64.5\%) & $64(65.3 \%)$ \\
\hline
\end{tabular}

${ }^{*}$ Patients were treated by camrelizumab plus gemcitabine and oxaliplatin from the NCT03486678 trial.

CHOL, cholangiocarcinoma; GBC, gallbladder cancer; ECOG PS,

Eastern Cooperative Oncology Group Performance Status.

disease: the TCGA CHOL cohort, and the Shanghai GBC cohort (online supplemental figure 2). The KRAS and TP53 mutations were much more frequently observed in the advanced CHOL and GBC cohorts (figure 2C). Specifically, CHOL had a high incidence of KRAS-TP53 co-mutations (figure 2C), which had a favorable response to camrelizumab $(\mathrm{ORR}=75 \%$; figure 2D), while a lone KRAS mutation in CHOL was associated with poor prognosis (figure 2E) and immunotherapy outcomes (figure 2D and $\mathrm{F}$ ).

TMBs were similar between CHOL (median TMB $=6$ ) and GBC (6.5) in our cohort (online supplemental figure $3 \mathrm{~A})$. Interestingly, patients with advanced BTC and lower TMBs (the optimal cut-off values were determined using the survminer $\mathrm{R}$ package) had numerically longer OS without reaching a statistical significance than those with higher TMBs in the overall population (online supplemental figure $3 \mathrm{~B}$ ) and in patients treated with camrelizumab (online supplemental figure 3C).

Some genes were more frequently mutated in CHOL than in GBC (figure 3A). Importantly, these genes are involved with the KRAS signaling network indicated by the STRING database (figure 3B). Not surprisingly, the $\mathrm{TMB}$ of these genes, defined as K-TMB, was negatively correlated with ORR (figure 3C) and OS of immunotherapy (figure 3D). Subsequently, CHOL had numerically worse immunotherapy outcomes than GBC, but the difference was statistically non-significant (figure $3 \mathrm{E}$ and F). 


\section{Medical centers led by JPH}

Included 98 patients with targeted NGS:

1) GBC: 36 , CHOL: 62

2) OS available: 64

3) 1 -line $\mathrm{ICl}$ therapy in the NCT03486678 trial: 34 (all with OS, 33 with response data)

\section{Other cohorts:}

1) CHOL: TCGA, 36

2) GBC: Shanghai, 32

Figure 1 A flow diagram for the analysis process. JPH, Jiangsu Province Hospital; NGS, next-generation sequencing; CHOL, cholangiocarcinoma; GBC, gallbladder cancer; TCGA, The Cancer Genome Atlas; OS, overall survival; ICI, immune checkpoint inhibitor; LASSO, least absolute shrinkage and selection operator; GSEA, gene set enrichment analysis; IHC, immunohistochemistry; TME, tumor microenvironment.

A genetic signature predicts prognosis and immunotherapy outcomes

Univariate Cox models indicated 23 mutated genes that significantly impact the overall prognosis (figure 4A). Eleven mutated genes with the most powerful prognostic role were selected using the penalized Cox regression model with LASSO penalty (figure 4B). These genes, including APC, ARID1A, CCND1, DAXX, ERBB2, LRP1B, MED12, NRG1, PMS2, SMO, and TNFAIP3, composed a signature. Samples with at least one mutation in these genes were defined as the signature mutated subtype, which had a significantly worse OS than the signature wildtype subtype, not only in the overall population but also in the CHOL and GBC subgroups, respectively (figure 4C). 

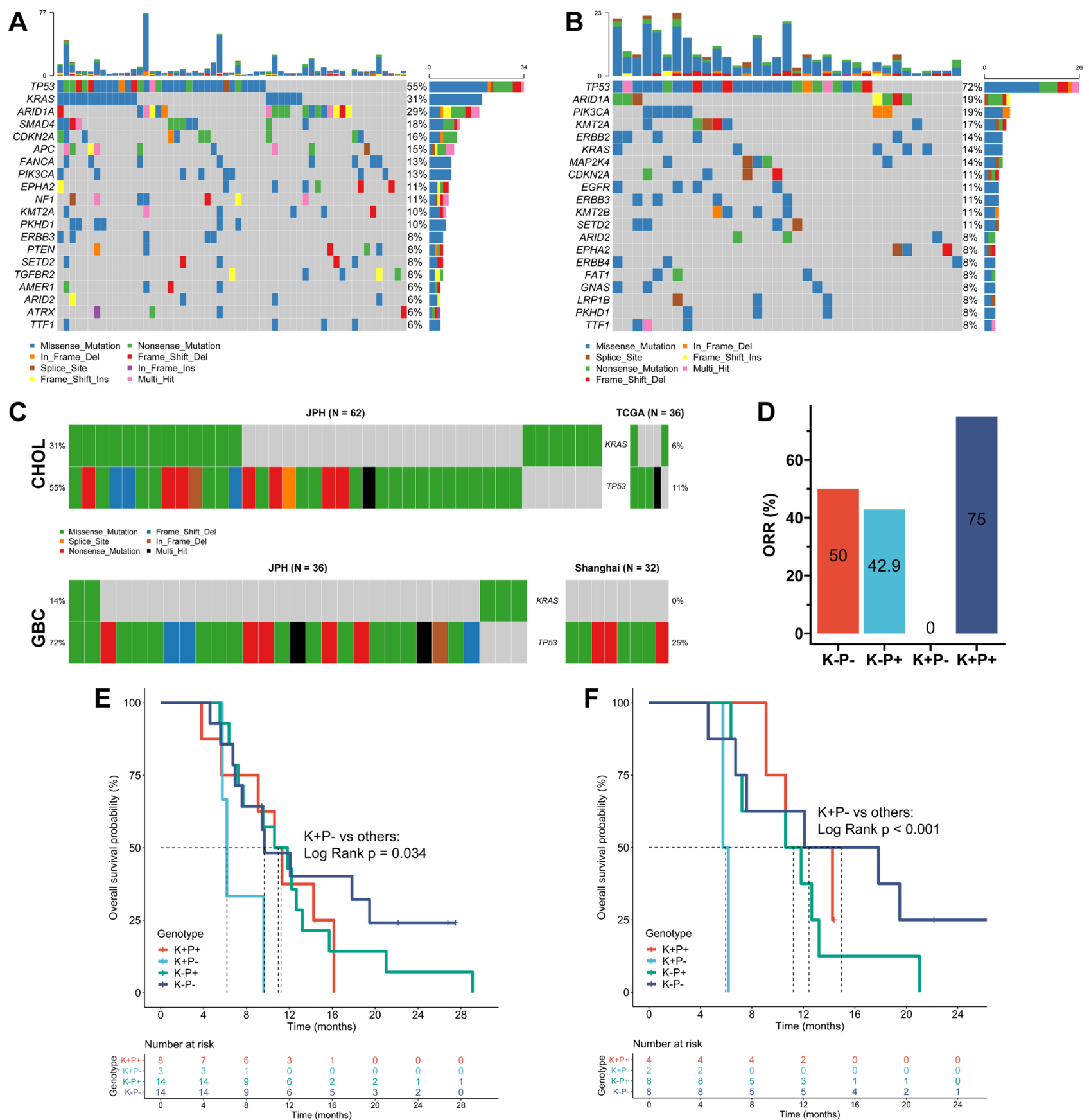

Figure 2 Genomic characteristics of advanced biliary tract cancer. (A and B) Waterfall plots showing the frequency and types of mutations found in the TOP20 mutated genes in advanced CHOL (A) and GBC (B). (C) Waterfall plots showing the frequency and types of mutations in KRAS and TP53 between the JPH cohort and other cohorts. (D) Overall response rate of immunotherapy stratified by both KRAS and TP53 mutational status in advanced CHOL. (E) Overall survival (OS) of all patients stratified by both KRAS and TP53 mutational status in advanced CHOL. (F) OS of patients treated by immunotherapy and stratified by both KRAS and TP53 mutational status in advanced CHOL. CHOL, cholangiocarcinoma; GBC, gallbladder cancer; TCGA, The Cancer Genome Atlas; JPH, Jiangsu Province Hospital.

Moreover, the prognostic impact of this signature classification was validated using the TCGA CHOL data and the Shanghai GBC data (figure 4D).

We further tested the impact of the signature classification on immunotherapy outcomes, and found that its wild-type subtype responded better to camrelizumab than its mutated subtype (66.7 vs $40 \%$; figure $5 \mathrm{~A}$ ) and also had significantly more favorable OS, not only in the overall immunotherapy population (figure $5 \mathrm{~B}$ ) but also in the CHOL (figure 5C) and GBC (figure 5D) subgroups, respectively.

Transcriptome features associated with the genetic signature Transcriptome data are available for the TCGA CHOL cohort, and their relationship to the genetic signature was analyzed. Differential gene expression (figure 6A) 

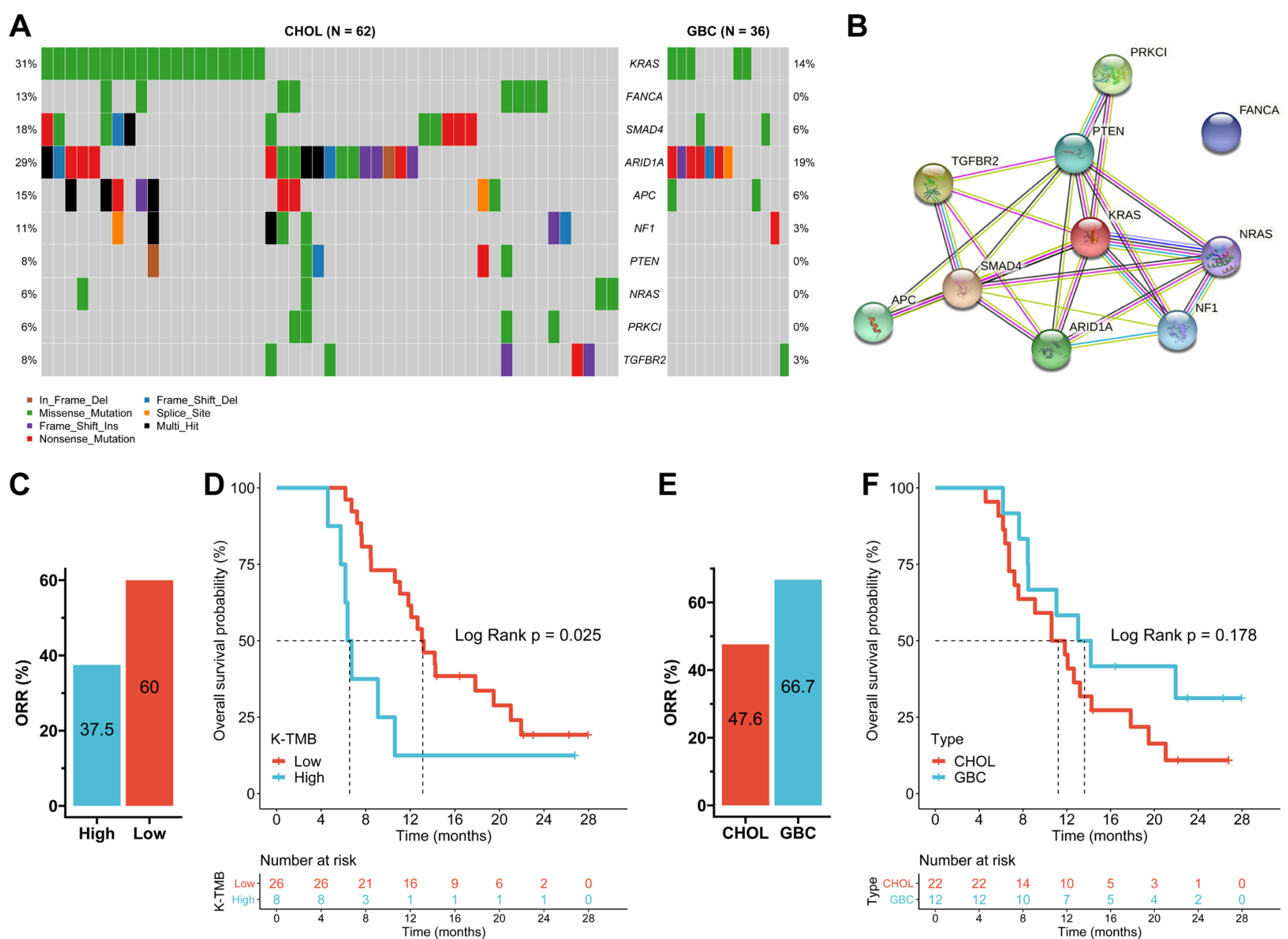

Figure 3 Genomic characteristics of advanced cholangiocarcinoma (CHOL) versus advanced gallbladder cancer (GBC). (A) Waterfall plots showing the frequency and types of mutations in genes that were more frequently mutated in $\mathrm{CHOL}$ than in GBC. (B) Proteins encoded by genes in a interact in the KRAS signaling network. (C) Overall response rate (ORR) after immunotherapy according to tumor mutation burden (TMB) of genes in a associated with KRAS signaling (K-TMB). (D) Overall survival (OS) of patients treated with immunotherapy and stratified by K-TMB. (E) Immunotherapy ORR of advanced CHOL and GBC. (F) OS of patients treated with immunotherapy in advanced $\mathrm{CHOL}$ and $\mathrm{GBC}$, respectively.

and subsequent gene set enrichment analysis (GSEA) showed that genes involved in immune-associated signaling or functions were significantly enriched in the signature mutated subtype (figure 6B). Specifically, this subtype is associated with primary immunodeficiency (figure 6C). However, T cell activation and differentiation may be facilitated by this subtype, while the TGF- $\beta$ signaling pathway, the driver of immune exclusion, may be inhibited (figure 6C). It is known that two primary mechanisms are involved in tumor immune evasion: dysfunction of infiltrating cytotoxic T lymphocytes (CTL) and CTL exclusion from tumors. ${ }^{12}$ Thus, we used the TIDE algorithm to calculate T cell dysfunction and exclusion scores and the merged TIDE Score which assessed tumor immune evasion. Using the median values as cut-offs, we found that the mutated subtype was more frequent to have high TIDE ( $70 \%$ vs $42.3 \%$ ) and high dysfunction scores ( $60 \%$ vs $46.2 \%$ ) but was less frequent to have a high exclusion score (40\% vs $53.8 \%)$ than the wild-type subtype (figure 6D). Besides, the abundance of infiltrating CD8 $+\mathrm{T}$ cells, calculated by the CIBERSORT algorithm based on transcriptome data, ${ }^{14}$ was slightly higher in the mutated subtype than that in the wild-type subtype ( $p=0.09$; figure $6 \mathrm{E}$ ). These findings were consistent with the GSEA results, suggesting that the mutated subtype has more severe tumor immune evasion and CTL dysfunction is a more popular underlying mechanism than CTL exclusion in this subtype.

We also used the TIDE algorithm to predict tumor responses to ICIs in the TCGA CHOL cohort. We verified the ORR superiority of the wild-type subtype than the mutated subtype ( $38.5 \%$ vs $20 \%$; figure $6 \mathrm{~F}$ ). In addition, in tumors whose responses were predicted as false, a high dysfunction score was more common in the mutated subtype $(75 \%$ vs $43.8 \%)$ but a high exclusion score was more common in the wild-type subtype $(81.3 \%$ vs $50 \%)$, indicating again the different immune evasion mechanisms between these two subtypes (figure 6G).

\section{TME associated with the genetic signature}

For TME evaluation, the infiltrated densities of several typical immune cells (figure 7A) and the expression 
A
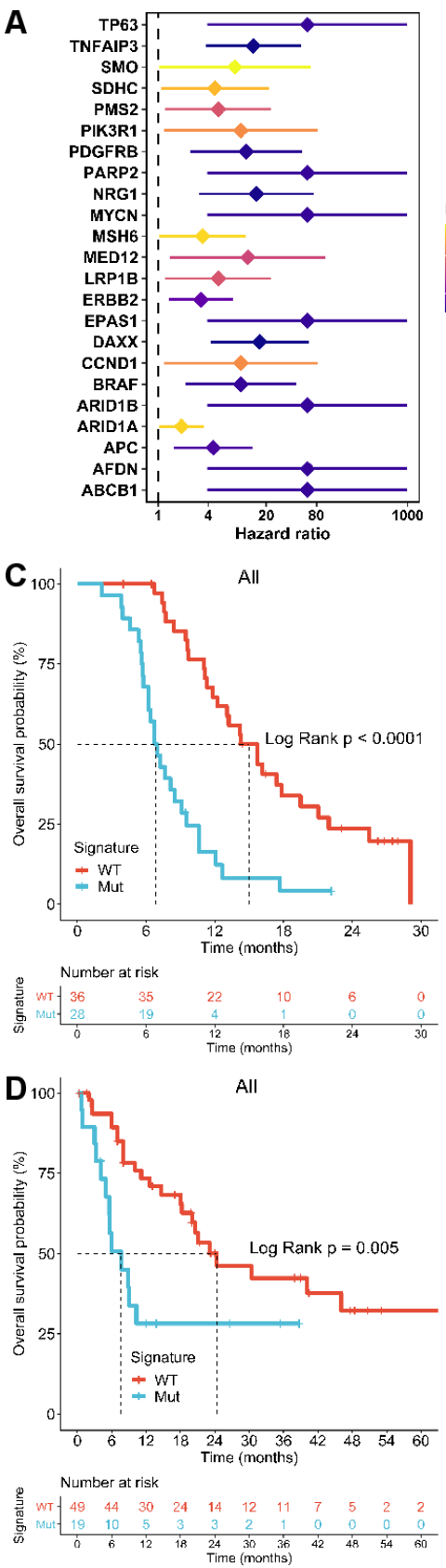
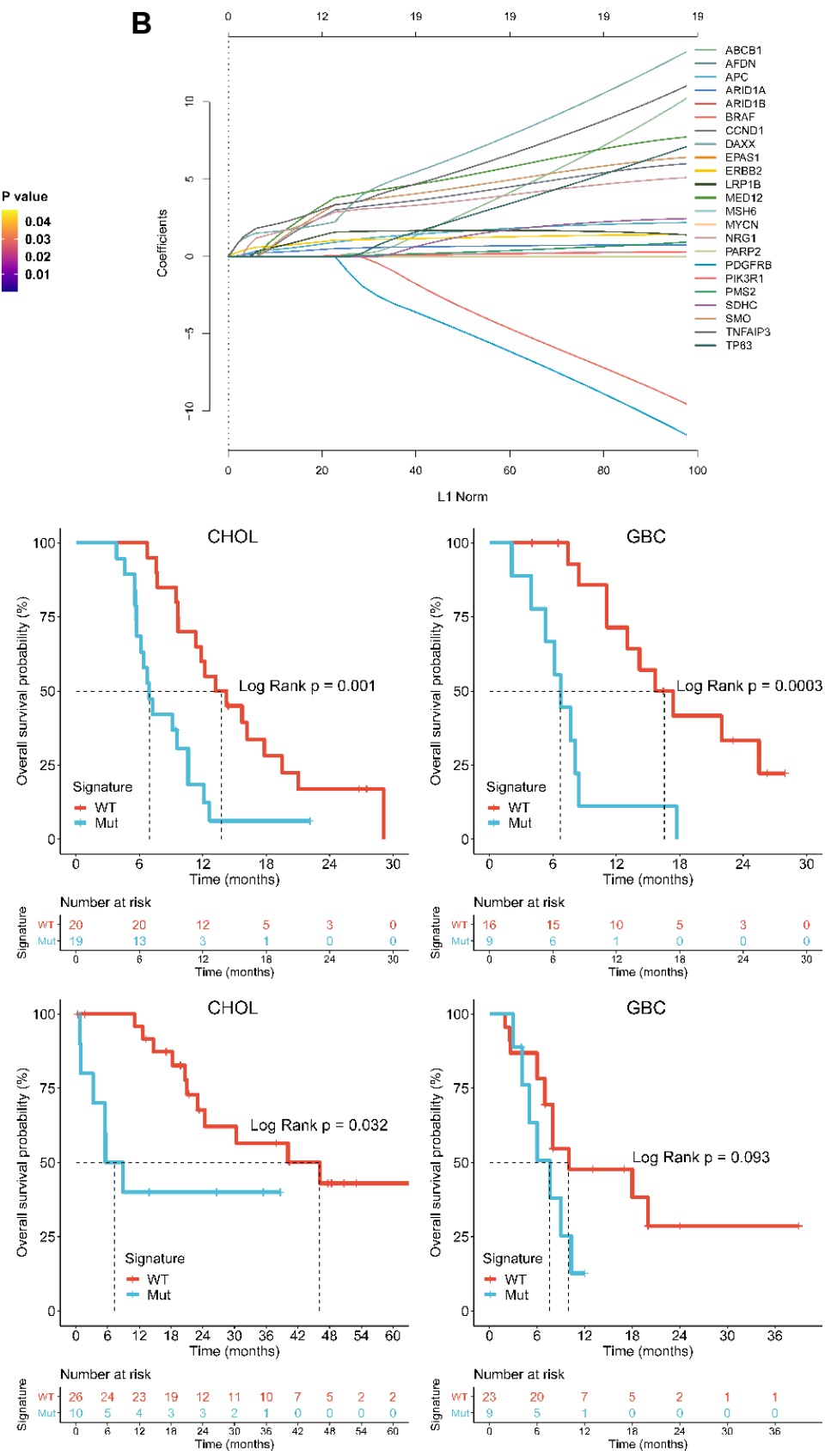

Figure 4 Genomic signature and prognosis. (A) HRs of genes with mutations that were significantly associated with overall survival (OS) in univariate COX models. (B) Least absolute shrinkage and selection operator (LASSO) coefficient profiles of the fractions of genes in a. (C) OS of patients in the JPH cohort according to mutation status of genomic signature constructed through the LASSO model in all patients, patients with CHOL, and patients with GBC, respectively. (D) OS of patients in the TCGA and Shanghai cohorts according to mutation status of the genomic signature in all patients, patients with CHOL, and patients with GBC, respectively. CHOL, cholangiocarcinoma; GBC, gallbladder cancer; TCGA, The Cancer Genome Atlas; WT, wild-type; mut, mutated type; JPH, Jiangsu Province Hospital.

of PD-L1 (figure 7B) were detected by IHC in $30 \mathrm{BTC}$ tissues from the NCT03486678 trial. Because immune cells infiltrating only tumor parenchyma are cytotoxic, the cells in tumor parenchyma and stroma were quantified separately. Unsupervised clustering, based on the density of infiltrating immune cells in tumor parenchyma, identified two clusters (figure 7C). The cluster 1 (C1) had more infiltrating NK cells including CD56bright and CD56dim subtypes than the cluster 2 (C2), indicating that $\mathrm{C} 1$ and $\mathrm{C} 2$ owned different TME status. More tumors in C1 were PD-L1-positive and the signature mutated subtype (figure 7D). Importantly, the ORR and OS of immunotherapy in the signature wild-type subgroup could be further stratified by the clusters: C1 had a non-significant higher ORR $(85.7 \%$ vs $45.5 \%, \mathrm{p}=0.088$; figure $7 \mathrm{E}$ ) and a significantly longer OS ( $p=0.04$; figure $7 F$ ) than C2. Similar results were not found in the mutated subtype (figure $7 \mathrm{E}, \mathrm{F}$ ).

To validate our observations, we applied the TMEscore algorithm, which was also based on immune infiltration 
A
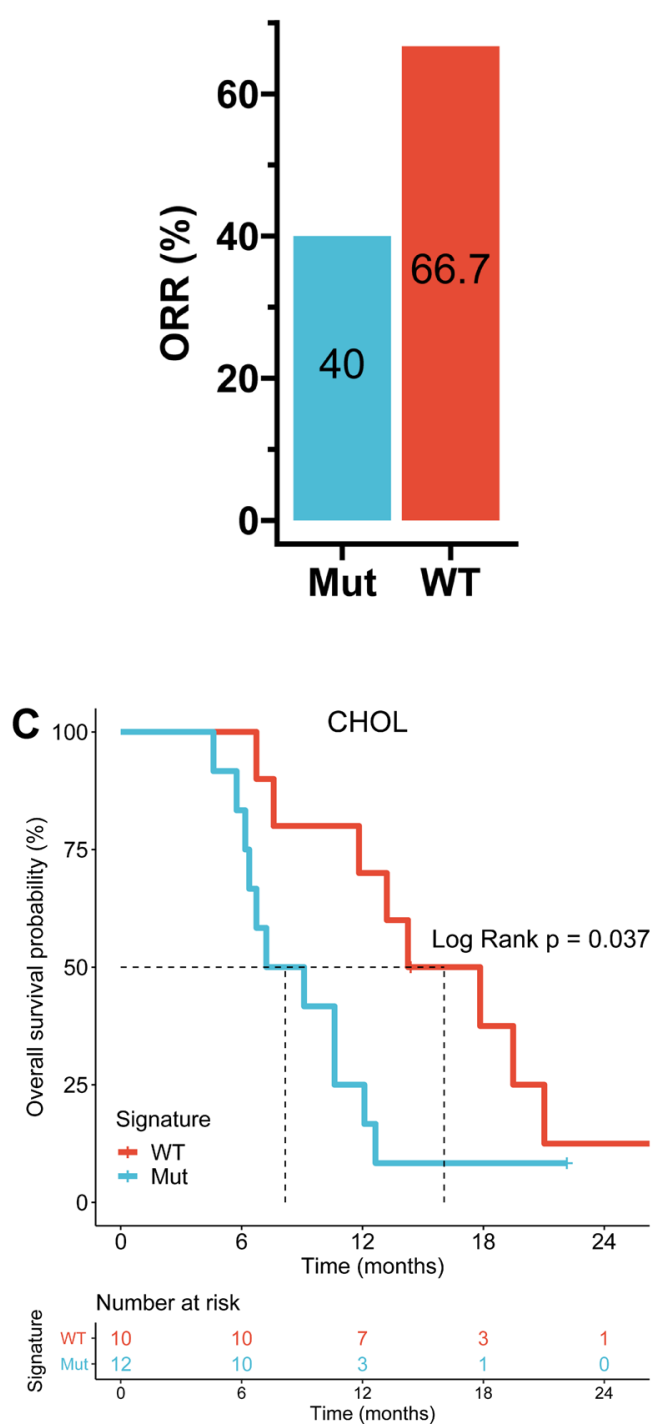
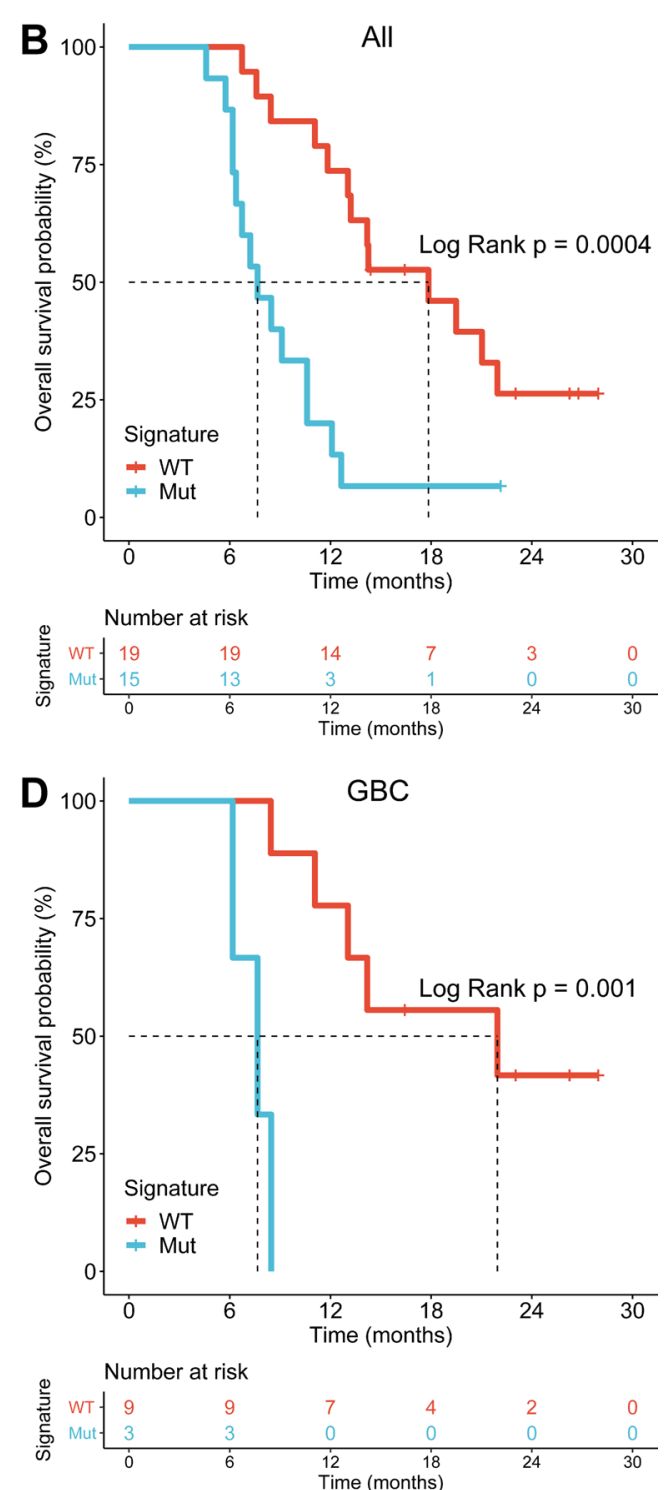

Figure 5 Genomic signature and immunotherapy outcomes. (A) Overall response rate of immunotherapy according to mutation status of the genomic signature. (B-D) Overall survival of immunotherapy according to mutation status of the genomic signature in all patients (B), patients with $\mathrm{CHOL}(\mathrm{C})$, and patients witj $\mathrm{GBC}(\mathrm{D})$, respectively. $\mathrm{CHOL}$, cholangiocarcinoma; $\mathrm{GBC}$, gallbladder cancer; mut, mutated type; WT, wild-type.

phenotypes ${ }^{13}$ to evaluate TME status as favorable TME (fTME) and poor TME (pTME) in the TCGA CHOL cohort, using the median TMEscore as a cut-off. ${ }^{11}$ Similarly to the results from the NCT03486678 trial, fTME tumors in the signature wild-type subgroup had better ORR predicted by TIDE algorithm $(63.6 \%$ vs $20 \%, p=0.024)$ and numerically longer OS $(p=0.29)$ than $p T M E$ tumors (online supplemental figure $4 \mathrm{~A}$ and $\mathrm{B}$ ). No such findings existed in the signature mutated subtype (online supplemental figure 4A and $\mathrm{C}$ ). Together, TME evaluation further improved the stratification of immunotherapy outcomes in the signature wild-type subset but not in the mutated subset, suggesting again immune dysfunction in the mutated subset.

\section{DISCUSSION/CONCLUSIONS}

In this study, we investigated the genomic mutation profile of advanced BTC, and found that DNA changes were associated with prognosis and first-line immunotherapy outcomes. Specifically, a classification based on a genetic signature was developed to improve the selection of patients with BTC for first-line immunotherapy. The mutated subtype of this classification may be characterized by an impaired antitumor immunity. Moreover, the combination of this classification and TME evaluation further improved the stratification of clinical outcomes.

We showed the enormous genomic heterogeneity between our advanced disease cohort and the early stage TCGA CHOL and Shanghai GBC cohorts. In particular, we reported the highest mutation incidences for KRAS and TP53, two genes commonly mutated in BTC with crucial roles in its pathogenesis, indicating that the frequency of some driver alterations may be dependent on the disease stage of BTC. 


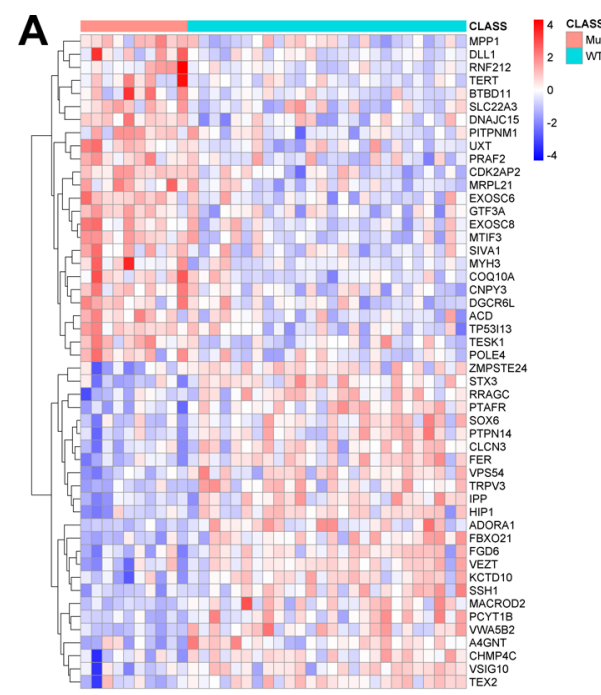

A

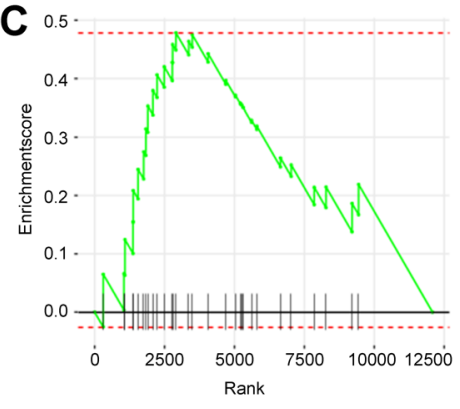

Primary_immunodeficiency

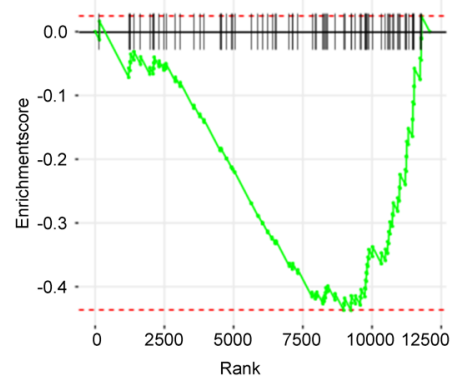

TGF-beta_signaling_pathway
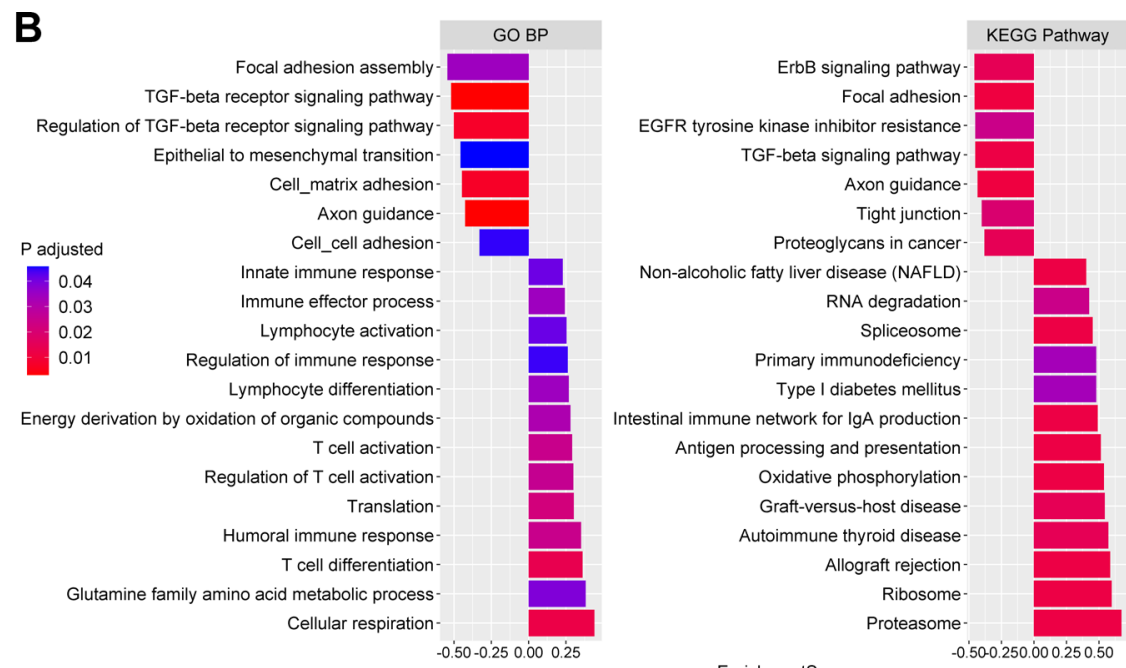

EnrichmentScore

D
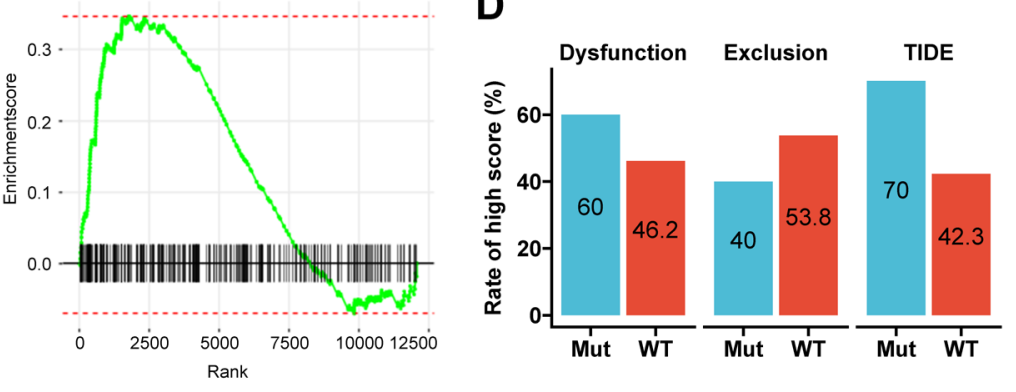

E

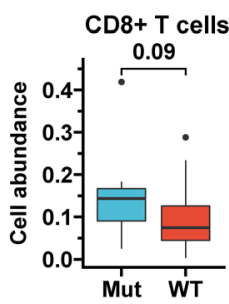

$\mathbf{F}$
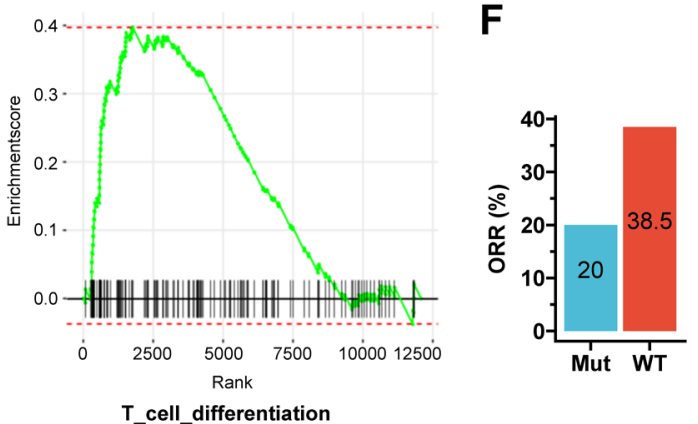

G

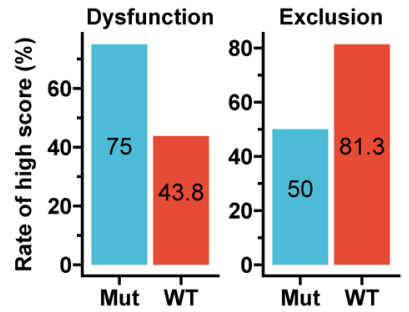

Figure 6 Transcriptome features associated with the genomic signature. (A) Heat map of TOP50 genes differentially expressed between the signature mutated and wild-type subgroups in the TCGA CHOL cohort. (B) Gene set enrichment analysis (GSEA) of the signature mutated subtype versus wild-type subtype. Selected entries of biological process (BP) in gene ontology (GO) and pathways in Kyoto Encyclopedia of Genes and Genomes (KEGG) are shown. (C) Enrichment plots of GSEA for several crucial GO terms or KEGG pathways. (D) T cell dysfunction, T cell exclusion and TIDE Scores according to the signature subtypes. (E) The abundance of infiltrating CD8 $+T$ cells according to the signature subtypes. $(F)$ The predicted responses to immune checkpoint inhibitors (ICls) by the TIDE algorithm according to the signature subtypes. (G) T cell dysfunction and exclusion scores according to the signature subtypes in tumors predicted to fail in ICl therapy. mut, mutated type; WT, wild-type; ORR, objective response rate.

We further observed that KRAS and TP53 frequently co-occurred in CHOL. It is known that KRAS-TP53 co-mutations promote an inflamed TME in lung adenocarcinoma, and may be of clinical benefit for immunotherapy. ${ }^{15}$ The underlying mechanisms are associated with deficient DNA damage repair caused by TP53 mutations, thus giving rise to increased genetic mutations and immunogenicity of cancer cells. ${ }^{16}$ Meanwhile, KRAS-TP53 co-mutations increase PD-L1 expression and infiltrating immune cells than KRAS-only or TP53-only mutations, indicating a synergistic effect. ${ }^{16}$ Similarly, we found that patients with KRAS-TP53 co-mutations had the highest ORR following immunotherapy among patients with CHOL with varied combinations of KRAS and TP53 mutation status. Moreover, patients with KRAS-only mutations had the worst prognosis and immunotherapy outcomes. These results indicate the effect of genomic alterations on the clinical heterogeneity of CHOL.

We compared the genomic alterations found in $\mathrm{CHOL}$ and GBC. At first, no TMB difference was found between 

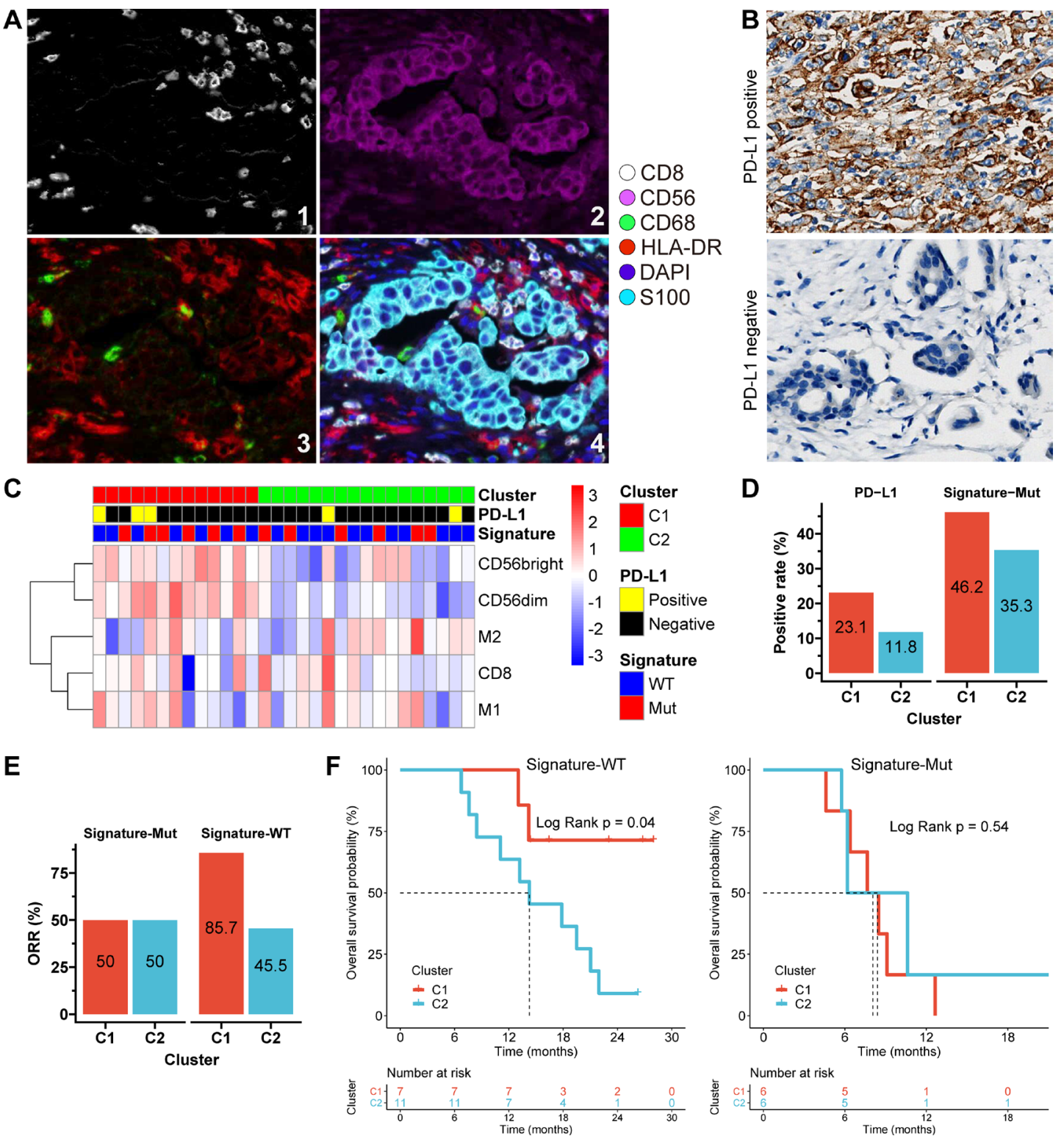

Figure 7 Tumor microenvironment (TME) associated with the genomic signature. (A) Typical micrographs for multiplexed immunohistochemistry (mIHC) staining of surface biomarkers of immune cells in tissues, at 200× magnification. 1, CD8; 2 , CD56; 3, CD68 (green) and HLA-DR (red); 4, the reconstructed image for all surface biomarkers. (B) Typical micrographs of programmed death ligand-1 (PD-L1)-positive and negative tumors, at 200x magnification. (C) Clustering based on the density of infiltrating immune cells in tumor parenchyma identified cluster 1 (C1) and cluster 2 (C2). (D) The positive rate of PD-L1 expression and the signature mutations according to clusters. ( $E$ and $F$ ) ORR $(E)$ and overall survival $(F)$ of immunotherapy according to the clusters and the genomic signature typing. ORR, objective response rate; mut, mutated type; WT, wild-type.

them. Interestingly, high TMB correlated with poor prognosis and immunotherapy outcomes in BTC, which verified a recent report that high TMB tumors exhibited lower immunotherapy efficacy than low TMB tumors in some tumor types. ${ }^{17}$ Furthermore, we found that in CHOL, more mutations occurred in KRAS and other genes encoding proteins which interact with KRAS protein. Immunotherapy outcomes were better stratified using the TMB of these genes (K-TMB) than the overall TMB, and high K-TMB correlated with worse outcomes. Subsequently, a similar difference in immunotherapy outcomes was observed between CHOL and GBC, although which was not statistically significant. These findings stress that
KRAS signaling can define BTC subgroups with distinct therapeutic vulnerabilities and may play a more important role in CHOL than in GBC.

Different from KRAS mutations, which seemed to cooperate with other genetic mutations to impact clinical outcomes, mutations of some genes were individually associated with OS. A genetic signature was constructed based on these genes to include those with the most prominent prognostic role. Importantly, molecular subtyping using this signature successfully stratified the prognosis and immunotherapy outcomes of BTC. Through transcriptome analyses, we revealed that the signature mutated subtype was associated with more severe tumor immune 
evasion, which was dominated by CTL dysfunction rather than CTL exclusion, than the signature wild-type subtype. This was further validated by our TME analyses: immune infiltration-based clustering or TME scoring had a crosstalk only with the wild-type subtype to impact immunotherapy outcomes, indicating a lack of function for infiltrating immune cells in the mutated subtype. Interestingly, we identified that the wild-type subtype with fTME may be an advantageous population of immunotherapy.

This study has some limitations. First, our targeted approach to examine 416 cancer-related genes as opposed to whole genome or exome sequencing limits our ability to reveal a more comprehensive genomic landscape for advanced BTC. Second, the small sample size prevents us from comparing the genomic characteristics of CHOL arising in different locations, limits the statistical significance and even may cause biased results in some comparisons. Third, we failed to find a BTC cohort treated with immunotherapy to validate our results about immunotherapy outcomes. Moreover, the potentially abnormal immune reaction in the signature mutated subtype needs experimental evidences. Furthermore, the specific link of the signature typing with immunotherapy prognosis remains inconclusive because this typing was also prognostic in the overall JPH cohort which included patients that received chemotherapy alone. Finally, population heterogeneity, different sequencing platforms and mutation calling algorithms may limit the repeatability of our findings in other studies.

In conclusion, we revealed genomic heterogeneity in advanced BTC, which differed between CHOL and GBC, could predict prognosis and immunotherapy outcomes. It was feasible to develop a genomic signature to improve the stratification of clinical outcomes in advanced BTC, which may be further optimized by combining the genomic classification and TME evaluation. Further studies are needed to verify our findings.

\section{Author affiliations}

${ }^{1}$ Oncology, Jiangsu Province Hospital and Nanjing Medical University First Affiliated Hospital, Nanjing, Jiangsu, China

${ }^{2}$ Oncology, Jiangsu University Hospital, Jiangsu, China

${ }^{3}$ Oncology, Shanghai Jiao Tong University Medical School Affiliated Ruijin Hospital, Shanghai, China

${ }^{4}$ Biological Therapy, Eastern Hepatobiliary Surgery Hospital, Shanghai, China

${ }^{5}$ Key Laboratory of Carcinogenesis \& Translational Research, Peking University Cancer Hospital, Beijing, Beijing, China

${ }^{6}$ Abdominal Medical Oncology, Cancer Hospital of the University of Chinese

Academy of Sciences (Zhejiang Cancer Hospital), Hangzhou, China

${ }^{7}$ Oncology, Nanjing Medical University Second Affiliated Hospital, Nanjing, Jiangsu,

China

${ }^{8}$ Oncology, Zhejiang University School of Medicine Sir Run Run Shaw Hospital,

Hangzhou, Zhejiang, China

${ }^{9}$ Cancer Center, Jilin University First Hospital, Changchun, China

${ }^{10}$ Medical Oncology, Department of Gastrointestinal Cancer, Liaoning Cancer

Institute and Hospital, Shenyang, Liaoning, China

${ }^{11}$ Department of Medical Oncology, China Medical University First Hospital,

Shenyang, Liaoning, China

${ }^{12}$ Key Laboratory of Anticancer Drugs and Biotherapy of Liaoning Province, China

Medical University First Hospital, Shenyang, Liaoning, China

${ }^{13}$ Medical, Nanjing Geneseeq Technology Inc, Nanjing, China

${ }^{14}$ Medical, 3D Medicines Inc, Shanghai, China
${ }^{15}$ Hepatobiliary Center, The First Affiliated Hospital of Nanjing Medical University, Nanjing, China

${ }^{16}$ School of Public Health, Nanjing Medical University, Nanjing, Jiangsu, China

Acknowledgements The authors thank all the patients, their families and the institutions for supporting this study.

Contributors YS was responsible for the overall content as guarantor. XC, JL, JQ, YWS and YS designed the study. XC and DW wrote the first draft of the manuscript. XC, JL, JQ, Jun Zhou, JY, Yan Shi, ZW, HL, JC, JZ, YL, XL, and YS treated the patients and acquired data. DW, DZ, SC, XL, LZ, and YS analyzed the data. XC, DW, JL, JQ, Yan Shi and YS interpreted the data. XC, DW, JL, JQ and YS revised the manuscript.

Funding The study was funded by Jiangsu province 333 high level Talents Project $(X C)$, Innovation Funds From Chinese Society Of Clinical Oncology Youth Committee (Y-Young2019-060 to XC, and Y-Young2021-0107 to DW), Beijing Xisike Clinical Oncology Research Foundation ( Y-HR2019-0367 to XC ), the Advanced Health Talent of Six-One Project of Jiangsu Province (LGY2017069 to XC), Joint Research Project by Southeast University and Nanjing Medical University (3207027381 to XC), Project of Young Medical Talents in Jiangsu Province (QNRC2016829 to DW), 5123 Scholar Program of the Affiliated Hospital of Jiangsu University (51232017301 to DW), and China Postdoctoral Science Foundation (2021M693272 to DW).

Competing interests DZ, XL, LZ and YWS are the employees of Geneseeq Technology Inc. SC is the employee of 3D Medicines Inc. The remaining authors declare that they have no competing interests.

Patient consent for publication Not applicable.

Ethics approval The study was approved by relevant regulatory and independent ethics committee of the First Affiliated Hospital with Nanjing Medical University and done in accordance with the Declaration of Helsinki and the International Conference on Harmonization Good Clinical Practice guidelines. All patients provided written, informed consent before study entry.

Provenance and peer review Not commissioned; externally peer reviewed.

Data availability statement Data are available upon reasonable request. All data relevant to the study are included in the article or uploaded as supplementary information. All data relevant to the study that are not in the article and supplementary material are available from the corresponding author on reasonable request.

Supplemental material This content has been supplied by the author(s). It has not been vetted by BMJ Publishing Group Limited (BMJ) and may not have been peer-reviewed. Any opinions or recommendations discussed are solely those of the author(s) and are not endorsed by BMJ. BMJ disclaims all liability and responsibility arising from any reliance placed on the content. Where the content includes any translated material, BMJ does not warrant the accuracy and reliability of the translations (including but not limited to local regulations, clinical guidelines, terminology, drug names and drug dosages), and is not responsible for any error and/or omissions arising from translation and adaptation or otherwise.

Open access This is an open access article distributed in accordance with the Creative Commons Attribution Non Commercial (CC BY-NC 4.0) license, which permits others to distribute, remix, adapt, build upon this work non-commercially, and license their derivative works on different terms, provided the original work is properly cited, appropriate credit is given, any changes made indicated, and the use is non-commercial. See http://creativecommons.org/licenses/by-nc/4.0/.

\section{ORCID iDs}

Jianyi Zhao http://orcid.org/0000-0003-2171-0856

Yongqian Shu http://orcid.org/0000-0003-2103-0877

\section{REFERENCES}

1 Tariq N-U-A, McNamara MG, Valle JW. Biliary tract cancers: current knowledge, clinical candidates and future challenges. Cancer Manag Res 2019;11:2623-42.

2 Siegel RL, Miller KD, Jemal A. Cancer statistics, 2017. CA Cancer J Clin 2017;67:7-30.

3 Valle J, Wasan $\mathrm{H}$, Palmer $\mathrm{DH}$, et al. Cisplatin plus gemcitabine versus gemcitabine for biliary tract cancer. N Engl J Med 2010;362:1273-81.

4 Seesaha P-K, Wang K-X, Wang G-Q, et al. Current progress and future perspectives of immune checkpoint inhibitors in biliary tract cancer. Onco Targets Ther 2021;14:1873-82. 
5 Chen X, Wu X, Wu H, et al. Camrelizumab plus gemcitabine and oxaliplatin (GEMOX) in patients with advanced biliary tract cancer: a single-arm, open-label, phase II trial. J Immunother Cancer 2020;8:e001240.

6 Malenica I, Donadon M, Lleo A. Molecular and immunological characterization of biliary tract cancers: a paradigm shift towards a personalized medicine. Cancers 2020;12:2190.

7 Hoadley KA, Yau C, Hinoue T, et al. Cell-of-Origin patterns dominate the molecular classification of 10,000 tumors from 33 types of cancer. Cell 2018;173:291-304.

8 Li M, Zhang Z, Li X, et al. Whole-Exome and targeted gene sequencing of gallbladder carcinoma identifies recurrent mutations in the ErbB pathway. Nat Genet 2014;46:872-6.

9 Wang D, Wang N, Li X, et al. Tumor mutation burden as a biomarker in resected gastric cancer via its association with immune infiltration and hypoxia. Gastric Cancer 2021;24:823-34.

10 Zhang L, Han X, Shi Y. Association of MUC16 mutation with response to immune checkpoint inhibitors in solid tumors. JAMA Netw Open 2020;3:e2013201.
11 Duan R, Li X, Zeng D, et al. Tumor microenvironment status predicts the efficacy of postoperative chemotherapy or radiochemotherapy in resected gastric cancer. Front Immunol 2020;11:609337.

12 Jiang P, Gu S, Pan D, et al. Signatures of T cell dysfunction and exclusion predict cancer immunotherapy response. Nat Med 2018;24:1550-8.

13 Zeng $\mathrm{D}$, Wu J, Luo H, et al. Tumor microenvironment evaluation promotes precise checkpoint immunotherapy of advanced gastric cancer. J Immunother Cancer 2021;9:e002467.

14 Newman AM, Liu CL, Green MR, et al. Robust enumeration of cell subsets from tissue expression profiles. Nat Methods 2015;12:453-7.

15 Skoulidis F, Heymach JV. Co-occurring genomic alterations in non-small-cell lung cancer biology and therapy. Nat Rev Cancer 2019;19:495-509.

$16 \mathrm{Gu} \mathrm{M}, \mathrm{Xu}$ T, Chang P. KRAS/LKB1 and KRAS/TP53 co-mutations create divergent immune signatures in lung adenocarcinomas. Ther Adv Med Oncol 2021;13:17588359211006950.

17 McGrail DJ, Pilié PG, Rashid NU, et al. High tumor mutation burden fails to predict immune checkpoint blockade response across all cancer types. Ann Oncol 2021;32:661-72. 